\title{
Backward-ion acceleration in critical density plasmas
}

Hideaki Habara ( $\nabla$ habara@eei.eng.osaka-u.ac.jp )

Osaka University https://orcid.org/0000-0002-7697-6830

Tomoyuki Iwawaki

Osaka University

Shinnosuke Nakagutchi

Osaka University

Yuto Uematsu

Osaka University

Kiyoshi Aizawa

Osaka University

Yuya Enmei

Osaka University

Sophie Baton

LULI

Christopher Rousseaux

CEA

Sophia Chen

LULI

Julien Fuches

LULI

Wigen Nazarov

School of Chemistry, University of St. Andrews

Michael MacDonald

SLAC National Accelerator Laboratory https://orcid.org/0000-0002-6295-6978

Ryosuke Kodama

Graduate School of Engineering

Kazuo Tanaka

Graduate School of Engineering

Physics Article

Keywords: ion acceleration, critical density plasmas, laser-accelerated ions 
Posted Date: June 7th, 2021

DOl: https://doi.org/10.21203/rs.3.rs-514850/v1

License: (c) (i) This work is licensed under a Creative Commons Attribution 4.0 International License. Read Full License 


\title{
Backward-ion acceleration in critical density plasmas
}

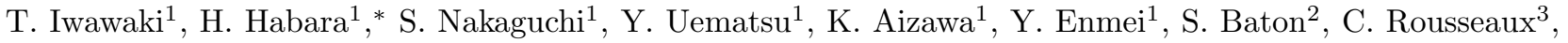 \\ S.N. Chen ${ }^{2,4}$, J. Fuchs ${ }^{2,4}$, W. Nazarov ${ }^{5}$, M.J. MacDonald ${ }^{6,7}$, R. Kodama ${ }^{1,8}$, and K.A. Tanaka ${ }^{1,9}$ \\ ${ }^{1}$ Graduate School of Engineering, Osaka University, \\ Yamada-oka, 2-1, Suita, Osaka 565-0871, Japan \\ ${ }^{2}$ LULI, CNRS-CEA-Ecole Polytechnique-Sorbonne Universit, 91128 Palaiseau Cedex France. \\ ${ }^{3}$ CEA, DAM, DIF, F-91297 Arpajon, France. \\ ${ }^{4}$ Institute of Applied Physics, 46 Ulyanov Street, 603950 Nizhny Novgorod, Russia. \\ ${ }^{5}$ School of Chemistry, University of St. Andrews, \\ North Haugh, St. Andrews, Fife KY16 9ST, Scotland, UK \\ ${ }^{6}$ SLAC National Accelerator Laboratory, Menlo Park, CA, USA \\ ${ }^{7}$ University of Michigan, Ann Arbor, MI, USA \\ ${ }^{8}$ Institute of Laser Engineering, Osaka University, \\ Yamada-oka, 2-6, Suita, Osaka 565-0871, Japan and \\ ${ }^{9}$ Extreme Light Infrastructure: Nuclear Physics (ELI-NP), \\ Str. Reactorului no.30, P.O.BOX MG-6, Bucharest - Magurele, Romania
}

(Dated: May 18, 2021)

\begin{abstract}
Laser-accelerated ions are widely attractive for many fields as a compact and low-cost accelerator. For the application to medical treatment, it is necessary to not only enhance the maximum acceleration energy but also improve beam stability and quality. In this study, we demonstrate a new ion acceleration mechanism using a uniform critical density plasma that yields very high ion energy despite the existence of a laser prepulse. The maximum proton energy in the experiment was approximately $18 \mathrm{MeV}$ accelerated by the laser pulse of $3 \times 10^{19} \mathrm{~W} / \mathrm{cm}^{2}$ focused intensity under the conditions of the maximum prepulse contrast ratio of $10^{-3}$. Further, heavier particles such as carbon or oxygen present in the plasma were accelerated using the same acceleration field. In addition, a self-created magnetic field in the plasma significantly improved emission divergence.
\end{abstract}

\section{INTRODUCTION}

Energetic ion beams accelerated by an ultra-intense laser (UIL) are commonly expected to have the potential to yield very low emittance and short burst durations. A very large accelerating field $\left(>10^{12} \mathrm{Vm}^{-1}\right)$ causes ions to be accelerated to over mega-electron-volt energies within micron length scales. These features enable us to envision ultra-compact accelerators for various applications such as medical treatment or neutron generators. Over the past decades, energetic ion beams accelerated by an UIL have been extensively studied; from a physical point of view, ions are best accelerated by a sheath electric field generated on the backside of a thin foil target; this is called target normal sheath acceleration (TNSA) [15]. Recently, the acceleration of ions in a very thin target using a very strong photon pressure of laser light (radiation pressure acceleration; RPA) $[6,7]$ was demonstrated. Further, the laser light was shown to re-accelerate the ions to reach the Giga electron volt energy range after the laser passed through the thin foil owing to the relativistic effect [8]. High-energy ions are also obtained through Coulomb explosion or magnetic vortex acceleration in gas clusters $[9,10]$.

Although laser-driven ion acceleration is expected to generate ions with very high current and energy, a precursive laser pedestal or prepulse can change the target

* corresponding author; habara@eei.eng.osaka-u.ac.jp condition, which can degrade beam quality by breaking the thin foil or cluster targets. Many efforts have been invested to improve the temporal contrast of laser pulses [11-13]; this has resulted in achieving the best performance on the order of $10^{-12}$ in the nanosecond regime. However, the focal intensity of recent UIL pulses exceeds $10^{22} \mathrm{~W} / \mathrm{cm}^{2}$, and thus, the prepulse or the nanosecondpedestal remains sufficiently strong to ionise solid materials. Further, the installation of these devices can complicate the system and increase costs drastically.

In this paper, we propose a new ion-acceleration mechanism that is insensitive to such prepulses. Figure 1 shows the schematic of this acceleration mechanism based on the use of a uniform critical density plasma. When an UIL irradiates this target, the UIL penetrates the plasma because of the relativistic transparency effect. The electron temperature rises rapidly inside the laser pulse, which excites a force associated to the Ponderomotive potential $\left(-m_{e} c^{2} \nabla \gamma\right)$ between the heated and unheated regions [14]. In addition, the laser light pushes the plasma towards the propagation direction, thereby leading to an increase in the plasma pressure at the laser front. Then, the Ponderomotive potential and pressure gradient $\left(-\nabla p_{e}\right)$ at the pulse front accelerate electrons in the reflected light direction; this results in the formation of an electrostatic field which accelerates plasma ions in the backward direction. Simultaneously, the azimuthal magnetic field generated in the plasma by forward-accelerated electrons [15] reduces the beam divergence of the backward-accelerated ions.

The temperature of the uniform plasma already in- 
creases to several tens of electron volts [16], and therefore, the prepulses do not affect the plasma temperature considerably. Even if a prepulse exists, it is absorbed by the surface of the critical density plasma, thereby creating a thin preplasma in front of the target. However, the density of the preplasma is usually smaller than the relativistic critical density of the main pulse; therefore, the main pulse interacts directly with the main target. Using this experimental platform, we can obtain highquality ion beams even at a low-contrast laser pulse.

\section{ACCELERATION MODEL}

The equation of motion of electrons in a density gap can be represented by the relativistic fluid equation with the pressure term as

$$
\frac{\partial \mathbf{P}_{\mathbf{e}}}{\partial t}=\frac{e}{c} \frac{\partial \mathbf{A}}{\partial t}+e \nabla \phi-m_{e} c^{2} \nabla \gamma-\frac{\nabla p}{n_{e}} .
$$

where, $\mathbf{P}_{\mathbf{e}}$ is the electron momentum; $e$ is the electron charge; $c$ is the speed of light in vacuum; $\mathbf{A}$ is the vector potential of the laser light; $\phi$ is the electrostatic potential; $m_{e}$ is the electron mass; $\gamma$ is the Lorentz factor of electrons; $p$ is the plasma pressure, and $n_{e}$ is the plasma electron density. The terms on the right side indicates the force by laser, electrostatic field, Ponderomotive term, and pressure, respectively. Assuming stationary states [17], the slow frequency motion of electrons are balanced by the excitation of the electrostatic field, which becomes

$$
e \nabla \phi=m_{e} c^{2} \nabla \gamma+\frac{\nabla p}{n_{e}} .
$$

Equation 2 infers the Ponderomotive force and pressure at the laser front that emits electrons in the backward direction of the laser injection via the excited electrostatic potential; the plasma ions are then accelerated in the same direction. The maximum energy the ion can gain is given by

$$
W=Z e \int_{-\infty}^{\infty} \nabla \phi d x=3 Z m_{e} c^{2} \gamma_{0},
$$

where $\mathrm{Z}$ is the $\mathrm{Z}$-number of accelerated ions and $\gamma_{0}$ is the Lorentz factor of the plasma electrons in the plasma wall at the laser front.

The solid line in Fig. 2a indicates the maximum ion energy obtained using equation 3 for protons as a function of incident laser intensity. In this calculation, we assume $\gamma_{0}=\sqrt{(}\left(+a_{0}^{2} / 2\right)$ by using the normalised vector potential considering Doppler shift of the reflected light, $a_{0}=0.89 \times 10^{-9}\left(\sqrt{I_{\text {in }}}+\sqrt{R I_{\text {ref }}}\left(k_{\text {in }} / k_{\text {ref }}\right)\right)$, where $I_{\text {in }}, I_{\text {ref }}, k_{\text {in }}$, and $k_{\text {ref }}$ are the intensity and wave number of the incident and reflected light, respectively, and $R$ is the reflectivity. These parameters can be obtained from the laser and plasma parameters [18, 19]. We also show the other types of scaling from different acceleration mechanisms such as TNSA and experimental data, in Fig. 2a.

Based on this estimation, our model indicates that the maximum proton energies are several times larger than those estimated by the TNSA model. Further, our model predicts that it can obtain $200 \mathrm{MeV}$ protons, which are required for cancer therapy, when the laser intensity exceeds $3.7 \times 10^{21} \mathrm{~W} / \mathrm{cm}^{2}$. In our model, both incident and reflected lights contribute to the acceleration. Thus, backward ions can obtain higher acceleration energies compared to forward acceleration ions in the low-density foam target [30].

The static field in plasma can accelerate not only protons but also heavier ions. Fig. $2 \mathrm{~b}$ shows the ion spectra calculated using the 2D-PIC code for proton (red), carbon (green), and oxygen (blue) at $\tau_{\text {laser }}=300 \mathrm{fs}$ and $I_{\text {laser }}=2.5 \times 10^{19} \mathrm{~W} / \mathrm{cm}^{2}$ observed in front of the uniform plasma. In this calculation, the plasma simulates the foam material $\left(\mathrm{C}_{15} \mathrm{H}_{20} \mathrm{O}_{6}\right)$ used in the experiments; it is assumed that each ion is fully ionised. The observed maximum energy (85 MeV for carbon and $112 \mathrm{MeV}$ for oxygen) are smaller than the values calculated (120 and $160 \mathrm{MeV}$ ) using eq. 3, whereas the proton energy agrees well with the calculated value $(18 \mathrm{MeV})$. Lighter ions (protons) are precursory accelerated ahead of heavier ions (carbon or oxygen), and therefore, the electrostatic field is gradually neutralised, which results in the reduction of accelerated energies of heaver ions compared to that for the estimated values. However, our model indicates very efficient acceleration of heavy ions as shown by the number of heavier ions which is almost equivalent to that of the protons (proportion to the composition) in Fig. $2 \mathrm{~b}$. It may be also possible to accelerate more heavier ions by doping into the foam target.

\section{EXPERIMENTAL VALIDATION}

We conducted a laser experiment to validate this acceleration mechanism. Figure 3 shows the experimental setup. The target comprises a very low density (5 and $10 \mathrm{mg} / \mathrm{cc}$ ) plastic foam material inside a solid polyimide tube. One side of the opening of the tube is covered by a thin $\mathrm{Cu}$ foil $(700 \mathrm{~nm})$. A nanosecond-pulse laser irradiates on the foil to generate a homogenous critical density plasma $[15,16]$; the corresponding plasma density is approximately one and two times that of the critical density for $1.054 \mu \mathrm{m}$ laser light.

An intense laser light was then injected into the plasma from the opposite side of the tube. The pulse duration was changed from 0.3 to $1.5 \mathrm{ps}\left(I_{\text {laser }}=0.5-3.0 \times 10^{19}\right.$ $\left.\mathrm{W} / \mathrm{cm}^{2}\right)$. The accelerated ions in the backward direction were bent by the magnet pair in the horizontal direction, and then detected with a stack of radiochromic films (type HD-V2). The penetration of the intense laser light into the critical density plasma was confirmed by 
the measurement of strong Doppler spectral shift in the backscattered light using the same experimental setup and targets as in [31].

Figure 4 shows the experimental results obtained for the $5 \mathrm{mg} / \mathrm{cc}$ foam target. The pulse duration and intensity in this shot were $300 \mathrm{fs}$ and $2.5 \times 10^{19} \mathrm{~W} / \mathrm{cm}^{2}$, respectively. Figs. $4 \mathrm{a}$ and $\mathrm{b}$ indicate the 2nd and 10th RCF layer images. The ions bent by the magnet comes from the right to the left in this image, and clear horizontal lines are observed in each figure. Assuming these signals original from the protons, each layer indicates 2.8 and 9.8 $\mathrm{MeV}$ proton images. The vertical line plots are considered to analyse the data as shown in Fig. 4c. Although ions are bent in the horizontal direction, the vertical direction preserves the beam divergence. The estimated beam divergences are 12.7, 8.2, and $2.7^{\circ}$ (FWHM) for $2.8,9.8$, and $17.8 \mathrm{MeV}$ protons, respectively. This wellcollimated ion beam could be caused by the azimuthal magnetic field generated by the forward-accelerated electrons. In our previous study, we observed a $10^{\circ}$ beam divergence for the forward-accelerated electrons in the experiment using the same targets [15]. The 2D PIC calculation in this study reveals the formation of several 100 megaGauss magnetic fields around the plasma channel that help the collimation of the electrons. This magnetic field scatters backward electrons while converging the backward ions. This collimated backward ion beam is practically useful because it is easy to separate it from the laser axis using the magnet.

To confirm whether the signal on RCF comes from ions, the direction of magnet was reversed in some shots. No signal was observed even at the first layer of stack although backward electrons are recorded on the RCF. This relatively uniform background signal may be attributed to the small beam density of the backward electrons as they have a large beam divergence because of the azimuthal magnetic field. In addition, it was confirmed that the signal was not derived from neutrals or debris which are not affected by the magnetic field. Further, we tilted the target approximately $10^{\circ}$ to check the possibility of ions acceleration at the target surface caused by the sheath static field; however, the vertical position of the signals on the RCF image did not change. This result implies that ions are accelerated inside the plasma channel toward the back of the laser incident axis.

The maximum proton energies measured in the experiment are summarised in Fig. 5. Solid circles are experimental results for the $5 \mathrm{mg} / \mathrm{cc}$ foam target; the red lines represent the scaling calculated using eq. 3 ; the solid and broken lines indicate the results when the plasma electron densities are $1.0 n_{c}$ and $1.6 n_{c}$, respectively. The UIL was injected when plasma density became $1 n_{c}$ from the opposite side of the heating pulse. However, the propagation of UIL in the plasma may promote plasma ionisation up to $1.6 n_{c}$ (fully ionised plasma density of $5 \mathrm{mg} / \mathrm{cc}$ target). Therefore, the broken line seems to reproduce the experimental result more accurately, especially at the lower laser intensity. If the plasma density is relativistically over dense, no proton can be generated as the laser cannot propagate into the plasma. This is shown in the rapid drops of the scalings at the lower laser intensity.

Open circles represent the results from the 2D-PIC calculations. In the calculation, the UIL irradiates a critical density plasma using the same conditions as that in the experiment, and backward protons are observed at the front of the plasma. The simulated maximum proton energies reproduce the prediction obtained using eq. 3 and the experiments. Simultaneously, the PIC calculation well reproduces the proton spectra obtained in the experiment as shown in Fig. 5b. The solid line in the figure represents the accumulated proton spectra obtained at $5 \mu \mathrm{m}$ in front of the plasma. The open circles denote experimental data with cut-off energy of $15 \mathrm{MeV}$. The relative proton number in each layer was estimated from the calibration curve [32].

In Fig. 5a, the experimental results obtained using the $10 \mathrm{mg} / \mathrm{cc}$ foam target are shown as solid squares. At the highest UIL intensity, the maximum proton energy becomes close to $3.2 n_{c}$ probably because of the promotion of ionisation. Similar to the results of the $5 \mathrm{mg} / \mathrm{cc}$ foam target, the experimental results agree well with the estimated scaling. At the shot without long-pulse irradiation, the maximum proton energy for the $10 \mathrm{mg} / \mathrm{cc}$ foam target was considerably reduced to approximately $1 / 3\left(5.3 \mathrm{MeV}\right.$ at $\left.I_{\text {laser }}=2.3 \times 10^{19} \mathrm{~W} / \mathrm{cm}^{2}\right)$ of that with the long pulse. This result indicates the importance of plasma ionisation because the UIL will otherwise loose its energy during the propagation due to vacuum gap inside the foam.

Green triangles indicate the maximum energy protons accelerated in the TNSA using $20-\mu \mathrm{m} \mathrm{Au}$ foil targets. If the prepulse is sufficiently small, the maximum energy of protons generated from this target can exceed $12 \mathrm{MeV}$ at $I_{\text {laser }}=3 \times 10^{19} \mathrm{~W} / \mathrm{cm}^{2}[20]$. However, in this experiment, a prepulse exists with the maximum intensity of $10^{-3}$ of the main pulse arriving at $60 \mathrm{ps}$ earlier than the main pulse (see supplementary materials). Thus, the main pulse may interact with the preplasma, which can result in maximum energy of the generated proton to decrease to $2-4 \mathrm{MeV}$.

The effect of the prepulse can be limited in our acceleration model. We calculated an expected preformed plasma using a 2D MHD simulation to estimate this effect, and then, we simulated the maximum energy of the backward ions with this preformed plasma using a 2D PIC code. In the results, the prepulse with the intensity contrast of $10^{-3}$ that can generate the preformed plasma with a density scale between $n_{c}$ to $0.1 n_{c}$ is approximately $20 \mu \mathrm{m}$, which is relatively larger than the scale length without the prepulse of $2 \mu \mathrm{m}$. The PIC calculation shows that the presence of preformed plasma reduces the maximum ion energy to only $50 \%$ of the case without the prepulse (for more details, see the supplemental materials). This result indicates the robustness of this acceleration scheme compared to that of the TNSA or other acceleration schemes which require a very thin tar- 
get and a high contrast laser.

\section{SUMMARY}

In this paper, we discussed a very effective backwardion acceleration mechanism using a uniform critical density plasma. When UIL propagates into this relativistically under-dense plasma, the density and temperature gaps at the laser front excite the electrostatic field, which accelerates the electrons and subsequently the ions to the laser reflection direction. The maximum ion energies were found to be several times higher than those predicted by the TNSA mechanism. The observed ion beam showed a small beam divergence because of the collimation by the azimuthal magnetic field inside the plasma. The model presented here was found to be significantly robust against the prepulses or pedestal even at a contrast ratio of $10^{-3}$. Further, because the electrostatic field equally accelerates plasma ions, there is a possibility of using it as a particle source of high-current heavy ions.

\section{METHOD}

\section{Analytical accelerated ion energy}

We can obtain the accelerated ion energy by integrating eq. 3 in space. By substituting eq. 2 into eq. 3 , we get

$$
W=Z e \int \nabla \phi d x=Z \int\left(m_{e} c^{2} \nabla \gamma+\frac{\nabla p}{n_{e}}\right) d x .
$$

Here, pressure and temperature are given by

$$
\begin{aligned}
p & =n_{e} T_{e}, \\
T_{e} & =m_{e} c^{2}(\gamma-1) .
\end{aligned}
$$

Therefore, the pressure term can be rewritten as

$$
\begin{aligned}
\frac{\nabla p}{n_{e}} & =\frac{m_{e} c^{2}}{n_{e}}\left(\nabla\left(n_{e} \gamma\right)-\nabla n_{e}\right) \\
& =m_{e} c^{2}\left(\nabla \gamma+\frac{\nabla n_{e}}{n_{e}}(\gamma-1)\right) .
\end{aligned}
$$

When the intense laser propagates into the homogeneous plasma, the plasma inside the laser path is expelled by the strong Ponderomotive force, and a dense plasma wall is formed because of the pile-up in front of the laser pulse; this leads to large density and temperature $(=\gamma)$ gaps at the laser front similar to a step function (e.g., Fig.2a in ref[19]). In this case, the spatial distribution of density and $\gamma$ can be assumed as

$$
\begin{aligned}
\gamma(x) & =\frac{\gamma_{0}}{2}[\tanh (\alpha x)+1] \\
n_{e}(x) & =\frac{n_{0}}{2}[-\tanh (\beta x)+1],
\end{aligned}
$$

where $\gamma_{0}$ and $n_{0}$ indicate the $\gamma$ and density at the homogeneous plasma in front of laser pulse, respectively, and $\alpha$ and $\beta$ are parameters representing the spatial gradient that should be sufficiently large.

Substituting eqs. 5 and 6 into eq. 4, the integrated energy becomes

$$
\begin{aligned}
& W=2 Z e m_{e} c^{2}[\gamma]_{-\infty}^{\infty}+Z e m_{e} c^{2} \int_{-\infty}^{\infty} \frac{n_{e}^{\prime}}{n_{e}}(\gamma-1) d x \\
& =2 Z e m_{e} c^{2} \gamma_{0}+Z e m_{e} c^{2}\left\{\left[\frac{n_{e}}{n_{e}}(\gamma-1)\right]_{-\infty}^{\infty}\right. \\
& \left.-\int_{-\infty}^{\infty} n_{e}^{\prime}\left(\frac{\gamma-1}{n_{e}}\right)^{\prime} d x\right\} \\
& =3 Z e m_{e} c^{2} \gamma_{0} \\
& -Z_{e m} c^{2} \int_{-\infty}^{\infty} n_{e}^{\prime}\left(\frac{\gamma^{\prime} n_{e}-(\gamma-1) n_{e}^{\prime}}{n_{e}^{2}}\right) d x,(7)
\end{aligned}
$$

where the dash indicates the differential for the $x$ direction. Here, the differential for $\gamma$ and $n_{e}$ are

$$
\begin{aligned}
\gamma^{\prime}(x) & =\frac{\alpha \gamma_{0}}{2} \frac{1}{\cosh ^{2}(\alpha x)}, \\
n_{e}^{\prime}(x) & =-\frac{\beta n_{0}}{2} \frac{1}{\cosh ^{2}(\beta x)} .
\end{aligned}
$$

Therefore, if the plasma density at the laser front is sufficiently steep, it corresponds to $\alpha \rightarrow 0$ and $\beta \rightarrow 0$, then $\gamma^{\prime} \rightarrow 0$ and $n_{e}^{\prime} \rightarrow 0$, and $\gamma \rightarrow \gamma_{0}$ and $n_{e} \rightarrow n_{0}$ from eq. 6. Finally, we obtain eq. 3 .

\section{Experiment}

The experiment was performed at the ELFIE 100TW laser facility at LULI, Ecole Polytechnique, France, where both short-pulse and long-pulse laser beams are available. The target comprised very low plastic $\left(\mathrm{C}_{15} \mathrm{H}_{20} \mathrm{O}_{6}\right)$ foam materials with densities of 5 and 10 $\mathrm{mg} / \mathrm{cc}$ inside a solid polyimide tube. The length, inner diameter, and thickness of the tube were 300, 254, and $20 \mu \mathrm{m}$, respectively. One side of the opening of the tube was covered by a thin $\mathrm{Cu}$ foil $(700 \mathrm{~nm})$. A chirped (uncompressed) pulse irradiates on the foil to generate a homogenous critical density plasma over $200-\mu \mathrm{m}$ length. The wavelength, energy, focal intensity, and pulse duration of this pulse were $1.054 \mu \mathrm{m}, 60 \mathrm{~J}, 10^{14} \mathrm{~W} / \mathrm{cm}^{2}$, and $1 \mathrm{~ns}$, respectively. The formation of homogenous plasma was confirmed using X-ray radiography measurements [16]. The typical density of these plasmas were 1 
and 2 times of critical density for 5 and $10 \mathrm{mg} / \mathrm{cc}$ foam targets.

An intense laser light was then injected into the plasma from the opposite side of the tube. The pulse duration was changed from 0.3 to $1.5 \mathrm{ps}$, and the corresponding laser intensities were $0.5-3.0 \times 10^{19} \mathrm{~W} / \mathrm{cm}^{2}$. The ions accelerated backward for the laser incidence direction were bent by a $25-\mathrm{mm}$ length magnet pair with $0.45 \mathrm{~T}$ magnetic field strength located at $5 \mathrm{~mm}$ in front of the target. The backward-accelerated ions were detected with a stack of radiochromic films (type HD-V2) (more than 30 layers). The stack is attached at the exit of magnet, and therefore, the distance from TCC to RCF is $30 \mathrm{~mm}$. The distance between the magnets was $20 \mathrm{~mm}$, and therefore, the stack covers approximately $37^{\circ}$ in the full width as a detection angular window that is perpendicular to the magnetic field direction.

\section{D particle simulation}

Simulations were conducted with a $2 \frac{1}{2}$ fully electromagnetic PIC code FISCOF2 [33]. We considered a tar- get that consisted of fully ionised $\mathrm{C}_{15} \mathrm{H}_{20} \mathrm{O}_{6}$ plasma with totally $1 n_{c}$ electron density (for $1-\mu \mathrm{m}$ laser light). The length of the plasma is reduced to $60 \mu \mathrm{m}$ from $300 \mu \mathrm{m}$ in the experiment because we confirmed that the UIL with the highest intensity in this study does not penetrate the plasma. The transverse size is $50 \mu \mathrm{m}$, which is approximately five times larger than the pulse spot size $(10 \mu \mathrm{m}$ in FWHM). The s-polarised laser pulse is normally incident on the target with different peak intensities of 5,10 , and $30 \times 10^{18} \mathrm{~W} / \mathrm{cm}^{2}$. To minimise the calculation time, the pulse duration was fixed to $300 \mathrm{fs}$. For observing the backward-accelerated ions, a $100-\mu \mathrm{m}$ vacuum region was preserved in front of the plasma. The ion momentum was observed within $-100<x<70 \mu \mathrm{m}$, and the ion spectra were observed at $x=-5 \mu \mathrm{m}$ (the plasma was located between $0-60 \mu \mathrm{m})$.
[1] Clark, E.L., Krushelnick, K., Zepf, M., Beg, F.N., Tatarakis, M., Machacek, A., Santala, M.I.K., Watts, I., Norreys, P.A. \& Dangor, A.E.Energetic heavy-ion and proton generation from ultraintense laser-plasma interactions with solids. Phys. Rev. Lett. 85, 1654 (2000).

[2] Hatchett, S.P., Brown, C.G., Cowan,T.E., Henry, E.A., Johnson, J.S., Key, M.H., Koch, J.A., Langdon, A.B., Lasinski, B.F., Lee, R.W., Mackinnon, A.J., Pennington, D.M., Perry, M.D., Phillips, T.W., Roth, M., Sangster, T.C., Singh, M.S., Snavely, R.A., Stoyer, M.A., Wilks, S.C. \& Yasuike, K. Electron, photon, and ion beams from the relativistic interaction of Petawatt laser pulses with solid targets Physics of Plasmas 7, 2076 (2000).

[3] Snavely, R.A., Key, M.H., Hatchett, S.P., Cowan, T.E., Roth, M., Phillips, T.W., Stoyer, M.A., Henry, E.A., Sangster, T.C., Singh, M.S., Wilks, S.C., MacKinnon, A.J., Offenberger, A., Pennington, D.M., Yasuike, K., Langdon, A.B., Lasinski, B.F., Johnson, J., Perry, M.D. \& Campbell, E.M.High-Energy Proton Beams from Petawatt-Laser Irradiation of Solids. Phys. Rev. Lett. 85, 2945 (2000).

[4] Maksimchuk, A., Gu, S., Flippo, K., Umstadter, D. \& $\mathrm{Yu}$. Bychenkov, V. Forward Ion Acceleration in Thin Films Driven by a High-Intensity Laser. Phys. Rev. Lett. 84, 4108 (2000).

[5] Mora, P. Plasma expansion into vacuum. Phys. Rev. Lett. 90, 185002 (2003).

[6] Esirkepov, T., Borghesi, M., Bulanov, S.V., Mourou, G. \& Tajima, T. Highly efficient relativistic-ion generation in the laser-piston regime. Phys. Rev. Lett. 92, 175003 (2004).

[7] Kar, S., Kakolee, K.F., Qiao, B., Macchi, A., Cerchez, M., Doria, D., Geissler, M., McKenna, P., Neely, D., Osterholz, J., Prasad, R., Quinn, K., Ramakrishna, B.,
Sarri, G., Willi, O., Yuan, X.Y., Zepf, M. \& Borghesi, M. Ion Acceleration in Multispecies Targets Driven by Intense Laser Radiation Pressure. Phys. Rev. Lett. 109, 185006 (2012).

[8] Yin, L., Albright, B.J., Hegelich, B.M., Bowers, K.J., Flippo, K.A., Kwan, T.J.T. \& Fernandez, J.C. Monoenergetic and $\mathrm{GeV}$ ion acceleration from the laser breakout afterburner using ultrathin targets. Phys. Plasmas 14, 056706 (2007).

[9] Ditmire, T., Zweiback, J., Yanovsky, V.P., Cowan, T.E., Hays, G. \& Wharton, K.B. Nuclear Fusion from Explosions of Femtosecond Laser-Heated Deuterium Clusters. Nature 398, 489 (1999).

[10] Fukuda, Y., Faenov, A.Ya., Tampo, M., Pikuz, T.A., Nakamura, T., Kando, M., Hayashi, Y., Yogo, A., Sakaki, H., Kameshima, T., Pirozhkov, A.S., Ogura, K., Mori, M., Esirkepov, T.Zh., Koga, J., Boldarev, A.S., Gasilov, V.A., Magunov, A.I., Yamauchi, T., Kodama, R., Bolton, P.R., Kato, Y., Tajima, T., Daido, H. \& Bulanov, S.V. Energy Increase in Multi-MeV Ion Acceleration in the Interaction of a Short Pulse Laser with a Cluster-Gas Target. Phys. Rev. Lett. 103, 165002 (2009).

[11] Itatani, J., Faure, J., Nantel, M., Mourou, G.\& Watanabe, S. Suppression of the amplified spontaneous emission in chirped pulse-amplification lasers by clean highenergy seed-pulse injection. Opt. Commun. 148, 70-74 (1998).

[12] Minkovski, N., Petrov, G.I., Saltiel, S.M., Albert, O. \& Etchepare, J. Nonlinear polarization rotation and orthogonal polarization generation experienced in a single-beam configuration. J. Opt. Soc. Am. B 21, 1659-1664 (2004).

[13] Taurry, C., Quere, F., Geindre, J.-P., Levy, A., Ceccotti, T., Monot, P., Bougeard, M., Reau, F., D'oliveira, P., Audebert, P., et al. Plasma mirrors for ultrahigh intensity 
optics. Nature Phys. 3, 424-429 (2007).

[14] McKinstrie, C.J. \& DuBois, D.F. A covariant formalism for wave propagation applied to stimulated Raman scattering. Phys. Fluids 31, 278-287 (1988);

[15] Iwawaki, T., Habara, H., Baton, S., Morita, K., Fuchs, J., Chen, S., Nakatsutsumi, M., Rousseaux, C., Filippi, F., Nazarov, W. \& Tanaka, K.A. Collimated fast electron beam generation in critical density plasma. Phys. Plasmas 21, 113103 (2014).

[16] Chen, S.N., Iwawaki, T., Morita, K., Antici, P., Baton, S.D., Filippi, F., Habara, H., Nakatsutsumi, M., Nicolaï, P., Nazarov, W., Rousseaux, C., Starodubstev, M., Tanaka, K.A. \& Fuchs, J. Density and Temperature Characterization of Long-Scale Length, NearCritical Density Controlled Plasma Produced from UltraLow Density Plastic Foam. Sci. Report 6, 21495 (2016).

[17] Shen, B. \& Yu, M.Y, Relativistic channeling by intense laser pulse in overdense plasmas. Phys. Rev. E 68, 026501 (2003).

[18] Guerin, S., Mora, P., Adam, J.C., Heron A., \& Laval, G. Propagation of ultraintense laser pulses through overdense plasma layers. Phys. Plasmas 3, 2693 (1996).

[19] Iwawaki, T., Habara, H., Yabuuchi, T., Hata, M., Sakagami, H. \& Tanaka, K.A. Slowdown mechanisms of ultraintense laser propagation in critical density plasma. Phys. Rev. E 92, 013106 (2015).

[20] Fuchs, J., Antici, P., d'Humières, E., Lefebvre, E., Borghesi, M., Brambrink, E., Cecchetti, C.A., Kaluza, M., Malka, V., Manclossi, M., Meyroneinc, S., Mora, P., Schreiber, J., Toncian, T., Pèpin, H. \& Audbert, P. Laser-driven proton scaling laws and new paths towards energy increase. Nat. Phys. 2, 48 (2006).

[21] Hegelich, B.M., Pomerantz, I., Yin, L., Wu, H.C., Jung, D., Albright, B.J., Gautier, D.C., Letzring, S., Palaniyappan, S., Shah, R., Allinger, K., Herlein, R., Schreiber, J., Habs, D., Blakeney, J., Dyer, G., Fuller, L., Gaul, E., Mccary, E., Meadows, A.R., Wang, C., Ditmire, T. \& Fernandez, J.C. Laser-driven ion acceleration from relativistically transparent nanotargets. New J. Phys. 15, 085015 (2013).

[22] Passoni, M., Tikhonchuk, V.T., Lontano, M. \& Bychenkov, V.Yu. Charge separation effects in solid targets and ion acceleration with a two-temperature electron distribution. Phys. Rev. E 69, 026411 (2004).

[23] Nishiuchi, M., Fukumi, A., Daido, H., A. Sagisaka, Z. Li, Ogura, K., Orimo, S., Kado, M., Hayashi, Y., Mori, M., Bulanov, S.V.,Esirkepov, T., Nemoto, K., Oishi, Y., Nayuki, T., Fujii, T., Noda, A., Iwashita, Y., Shirai, T. \& Nakamura, S. The laser proton acceleration in the strong charge separation regime. Phys. Lett. A 557, 339 (2006).

[24] Robson, L., Simpson, P.T., Clarke, R.J., Ledingham, K.W.D., Lindau, F., Lundh, O., McCanny, T., Mora, P., Neely, D., Wahlström, C.-G., Zepf, M. \& McKenna, P. Scaling of Proton Acceleration Driven by PetawattLaser-Plasma Interactions. Nature Phys. 3, 58 (2007).

[25] Ogura, K., Nishiuchi, M., Pirozhkov, A.S., Tanimoto, T., Sagisaka, A., Esirkepov, T.Z., Kando, M., Shizuma, T., Hayakawa, T., Kiriyama, H., Shimomura, T., Kondo, S., Kanazawa, S., Nakai, Y., Sasao, H., Sasao, F., Fukuda, Y., Sakaki, H., Kanasaki, M., Yogo, A., Bulanov, S.V., Bolton, P.R., \& Kondo, K. Proton acceleration to $40 \mathrm{MeV}$ using a high intensity, high contrast optical parametric chirped-pulse amplification/Ti:sapphire hybrid laser system. Opt. Lett. 37, 2868 (2012) .
[26] Dollar, F., Reed, S.A., Matsuoka, T., Bulanov, S.S., Chvykov, V., Kalintchenko, G., McGuffey, C., Rousseau, P., Thomas, A.G.R., Willingale, L., Yanovsky, V., Litzenberg, D.W., Krushelnick, K. \& Maksimchuk, A. High-intensity laser-driven proton acceleration enhancement from hydrogen containing ultrathin targets. Appl. Phys. Lett. 103, 141117 (2013).

[27] Fritzler, S., Malka, V., Grillon, G., Rousseau, J.P. \& Burgy, F. Proton beams generated with high-intensity lasers: Applications to medical isotope production. Appl. Phys. Lett. 83, 3039 (2003).

[28] Spencer, I., Ledingham, K.W.D., McKenna, P., McCanny, T., Singhal, R.P., Foster, P.S., Neely, D., Langley, A.J., Divall, E.J., Hooker, C.J., Clarke, R.J., Norreys, P.A., Clark, E.L., Krushelnick, K., \& Davies, J.R. Experimental study of proton emission from 60-fs, 200$\mathrm{mJ}$ high-repetition-rate tabletop-laser pulses interacting with solid targets. Phys. Rev. E 67, 046402 (2003).

[29] Lee, K., Park, S.H., Cha, Y.-H., Lee, J.Y., Lee, Y.W., Yea, K.-H., \& Jeong, Y.U. Generation of intense proton beams from plastic targets irradiated by an ultraintense laser pulse. Phys. Rev. E 78, 056403 (2008).

[30] Willingale, L., Nagel, S.R., Thomas, A.G.R., Bellei, C., Clarke, R.J., Dangor, A.E., Heathcote, R., Kaluza, M.C., Kamperidis, C., Kneip, S., Krushelnick, K., Lopes, N., Mangles, S.P.D., Nazarov, W., Nilson, P. M., \& Najmudin, Z. Characterization of High-Intensity Laser Propagation in the Relativistic Transparent Regime through Measurements of Energetic Proton Beams. Phys. Rev. Lett. 102, 125002 (2009).

[31] Habara, H., Nakaguchi, S., Uematsu, Y., Baton, S.D., Chen, S.N., Fuchs, J., Iwawaki, T., M MacDonald Nazarov, W., Rousseaux C \& Tanaka, K.A. Propagation of intense short-pulse laser in homogeneous near-critical density plasmas. J. Phys. Conf. Ser. 717, 012019 (2016).

[32] Nurnberg, F. Schollmeier, M. Brambrink, E. Blazevic, A. Carroll, D.C. Flippo, K. Gautier, D.C. Geissel, M. Harres, K. Hegelich, B.M. Lundh, O. Markey, K. McKenna, P. Neely, D. Schreiber, J. \& Roth, M., Radiochromic film imaging spectroscopy of laser-accelerated proton beams, Rev. Sci. Instrum. 80 (2009) 033301.

[33] Sakagami, H. \&. Mima, K. Fast ignition simulations with collective PIC code. Proc. 2nd Int. Conf. Inertial Fusion Sciences and Applications, Kyoto, 2001, 380-383 (Elservier, 2002).

[34] Y.T. Li, Z.M. Sheng, Y.Y. Ma, Z. Jin, J. Zhang, Z.L. Chen, R. Kodama, T. Matsuoka, M. Tampo, K.A. Tanaka, T. Tsutsumi, T. Yabuuchi, K. Du, H.Q. Zhang, L. Zhang and Y.J. Tang, Phys. Rev. E 72 (2005) 066404.

[35] B. Fryxell, et al. Astrophys. J. Suppl. 131 (2000) 273334.

\section{ACKNOWLEDGEMENTS}

This work was fully supported by Grants-in-Aid for Scientific Research type A (Grant No. 22246122), type S (Grant No. 15H05751), and type C (Grant No. 18K03577) from the Japan Society for the Promotion of Science (JSPS). 


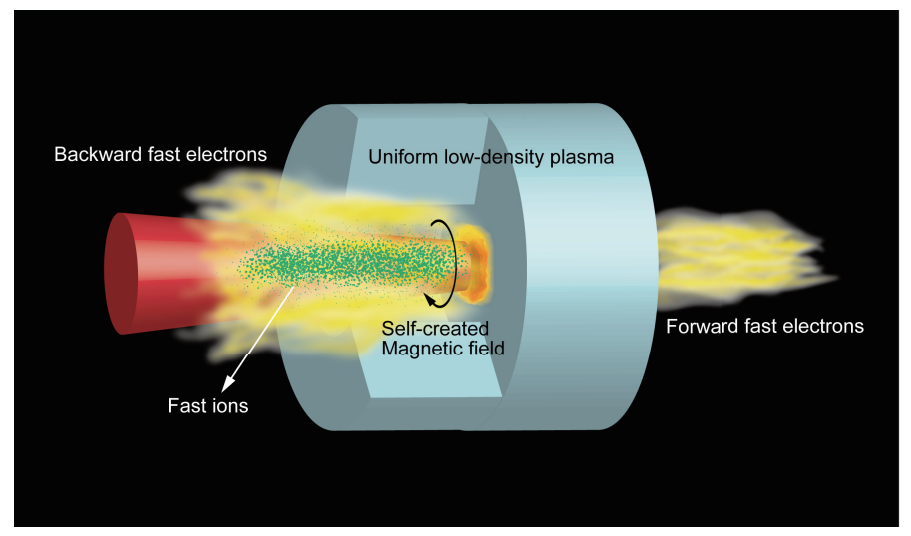

FIG. 1. Schematic image of the backward ion acceleration. When an ultra-high intensity laser irradiates a uniform critical density plasma, the density and temperature gap at the plasma channel front excites the electrostatic field. This field acts to accelerate the plasma ions to the backward direction. In addition, forward accelerated electrons inside the channel generate a magnetic field along the channel, which collimates backward accelerated ions.

\section{AUTHOR CONTRIBUTIONS}

$\mathrm{HH}$ and TI proposed the experiment and conceptualised the execution with KAT, JF, and SB. TI, HH, RK, and WN prepared the targets. The experiments were performed in collaboration with $\mathrm{HH}, \mathrm{TI}, \mathrm{SN}, \mathrm{YU}, \mathrm{SC}$, JF, $\mathrm{SB}, \mathrm{CR}$, and MM. IT and HH analysed the data. IT, KA, and YE performed the computer simulations. All authors discussed the results and arrived at the conclusions. $\mathrm{HH}$ drafted the paper and finalised it with contributions from other authors.

\section{COMPETING FINANCIAL INTERESTS}

The authors declare no competing financial interests. 


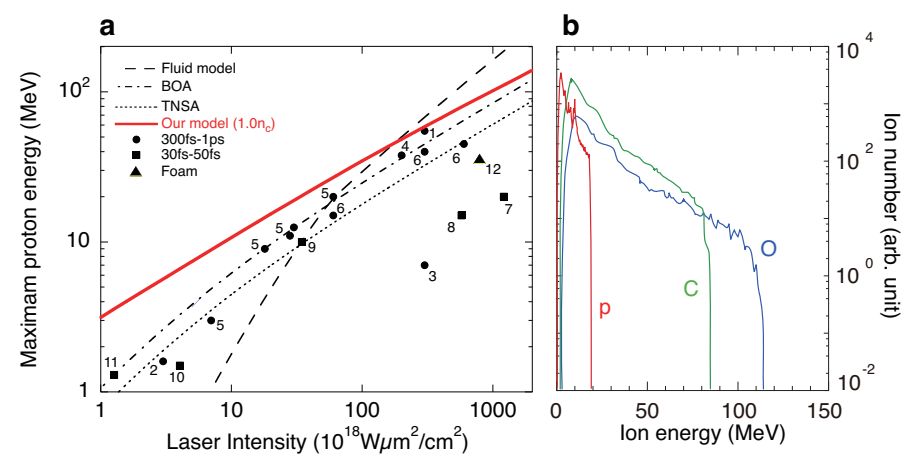

FIG. 2. a. Maximum proton energies. Each line present estimated scalings for each acceleration mechanisms; our model for $1 n_{c}$ plasma by eq. 3 (solid), fluid model $[5,20]$ (dashed), BOA [21] (dot-dashed), and TNSA model (dot) [22, 23]. Black circles and squares represent the experimental results from literatures in $300 \mathrm{fs}-1$ ps (labels 1 [2], 2 [4], 3 [7], 4 [21], 5 [20], and 6[24], and 30-60 fs (labels 7 [25], 8 [26], 9 [27], 10 [28], and 11 [29]), respectively. Solid triangle is the past result using low-density foam target [30]. b. Calculated ion spectra for proton (red), carbon (green), and oxygen (blue) for $\tau_{\text {laser }}=300 \mathrm{fs}$ and $I_{\text {laser }}=2.5 \times 10^{19} \mathrm{~W} / \mathrm{cm}^{2}$ observed at the plasma front.

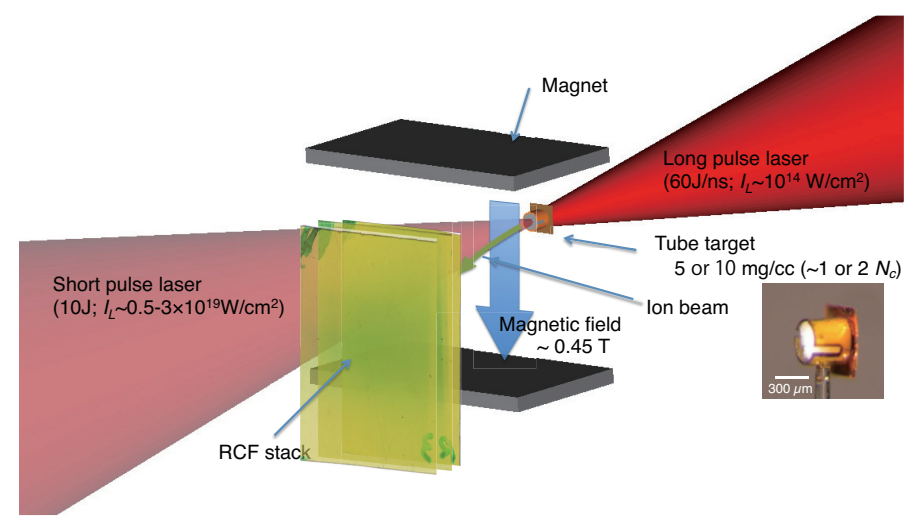

FIG. 3. Experimental setup. A low density form target in polyimide tube is pre-ionised by ns pulse laser. An intense short pulse laser $\left(300-1.5 \mathrm{ps} / 0.5-3.0 \times 10^{19} \mathrm{~W} / \mathrm{cm}^{2}\right)$ is then injected into the target from the opposite opening of the tube. The backward accelerated ions are bent by a magnet pair $(0.45 \mathrm{~T})$ and are detected with a RCF stack. 

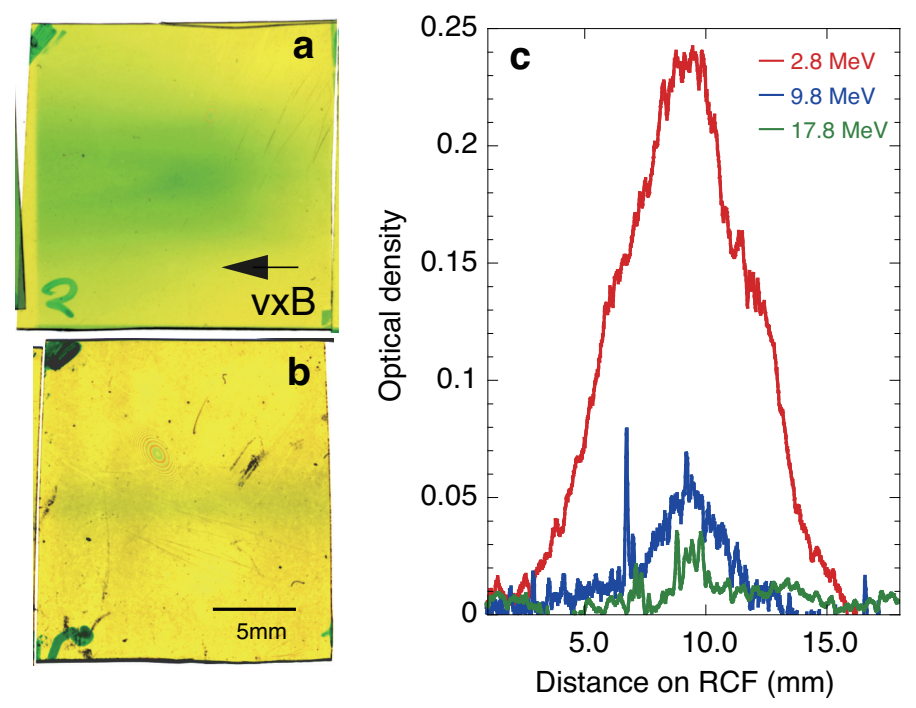

FIG. 4. a\&b. 2nd and 10th RCF layer (corresponding to 2.8 and $9.8 \mathrm{MeV}$ protons) images taken at $5 \mathrm{mg} / \mathrm{cc}$ foam target. The pulse duration and intensity in this shot were $300 \mathrm{fs}$ and $2.5 \times 10^{19} \mathrm{~W} / \mathrm{cm}^{2}$. The ions bent by the magnet come from right to left in this image. c. The vertical line profiles of each layer for $2.8,9.8$, and $17.8 \mathrm{MeV}$ protons, respectively. Beam divergence calculated from the signal width are 12.7, 8.2 and $2.7^{\circ}$ (FWHM).
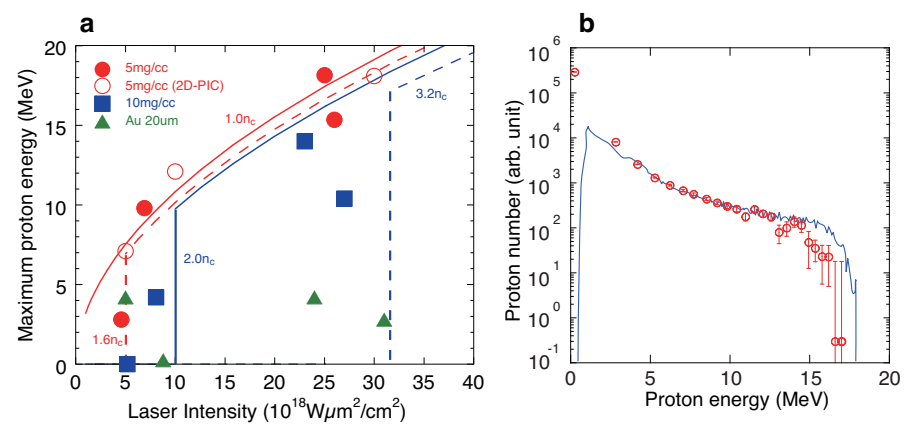

FIG. 5. a. Maximum proton energies obtained at the experiment. Solid and open circles are experimental and simulation results for $5 \mathrm{mg} / \mathrm{cc}$ foam target. Solid squares and triangles indicate the results of $10 \mathrm{mg} / \mathrm{cc}$ foam and $20-\mu \mathrm{m} \mathrm{Au}$ foil targets, respectively. Lines represent the scaling for different plasma density; $1 n_{c}$ (red solid), $1.6 n_{c}$ (fully ionised $5 \mathrm{mg} / \mathrm{cc}$ foam) (red dash), $2 n_{c}$ (blue solid), and $3.2 n_{c}$ (fully ionised $10 \mathrm{mg} / \mathrm{cc}$ foam) (blue dash). b. Proton energy spectra by experiment (circles) and simulation (line) for $\tau_{\text {laser }}=300 \mathrm{fs}$ and $I_{\text {laser }}=2.5 \times 10^{19} \mathrm{~W} / \mathrm{cm}^{2}$. 


\section{Supplementary Files}

This is a list of supplementary files associated with this preprint. Click to download.

- SIGUIDEDOA2100121.pdf 